Zbigniew Grande, Institute of Mathematics, Bydgoszcz Academy, Plac

Weyssenhoffa 11, 85-072 Bydgoszcz, Poland. e-mail:

grande@wsp. bydgoszcz.pl

\title{
ON POINTWISE, DISCRETE AND TRANSFINITE LIMITS OF SEQUENCES OF CLOSED GRAPH FUNCTIONS
}

\begin{abstract}
In this article we prove that if a function $f: X \rightarrow \mathcal{R}$ is the pointwise (discrete) [transfinite] limit of a sequence of real functions $f_{n}$ with closed graphs defined on complete separable metric space $X$ then $f$ is the pointwise (discrete) [transfinite] limit of a sequence of continuous functions. Moreover we show that each Lebesgue measurable function $f: \mathcal{R} \rightarrow \mathcal{R}$ is the discrete limit of a sequence of functions with closed graphs in the product topology $T_{d} \times T_{e}$, where $T_{d}$ denotes the density topology and $T_{e}$ the Euclidean topology.
\end{abstract}

We say that a function $f: X \rightarrow Y$, where $X$ and $Y$ are topological spaces, is a function with closed graph, if the graph of the function $f$, i.e. the set

$$
G(f)=\{(x, y) \in X \times Y ; y=f(x)\},
$$

is a closed subset of the product $X \times Y$.

Let $\mathcal{R}$ be the space of all reals with the Euclidean topology $T_{e}$. In the paper [10] Kostyrko proves that every function $f:\left(X, T_{X}\right) \rightarrow\left(\mathcal{R}, T_{e}\right)$ (shortly $f: X \rightarrow \mathcal{R})$ defined on a normal topological space $X$, with a closed graph is the limit of a sequence of continuous functions $f_{n}: X \rightarrow \mathcal{R}$, i.e. it is of the first class of Baire.

It is also obvious to observe that the uniform limit of a sequence of functions $f_{n}: X \rightarrow \mathcal{R}$ with closed graphs, has the closed graph ([6]).

In this article I prove that on a separable complete metric space $(X, \rho)$ the pointwise (resp. discrete) limit of a sequence of functions $f_{n}: X \rightarrow \mathcal{R}$ with closed graphs is the pointwise (resp. discrete) limit of a sequence of real continuous functions on $X$.

Key Words: Function with closed graph, discrete convergence, pointwise convergence, transfinite convergence, density topology.

Mathematical Reviews subject classification: 26A15, 54C30.

Received by the editors January 17, 2001

* Supported by Bydgoszcz Academy grant 2001 
Theorem 1. Let $(X, \rho)$ be a complete metric space. If a function $f: X \rightarrow \mathcal{R}$ is the pointwise limit of a sequence of functions $f_{n}: X \rightarrow \mathcal{R}$ with a closed graph then $f$ is of the first Baire class.

Proof. By Theorem 1 from [8] it suffices to prove that for every nonempty perfect set $A \subset X$ and for each positive real $\eta$ there is an open set $I$ such that

$$
I \cap A \neq \emptyset \text { and } \operatorname{osc}_{I \cap A} f<\eta .
$$

Let $A \subset X$ be a nonempty perfect set and let $\eta$ be a positive real. For each point $x \in A$ there is a positive integer $n(x)$ such that

$$
\left|f_{n}(x)-f(x)\right|<\frac{\eta}{8} \text { for } n>n(x) .
$$

For $n=1,2, \ldots$, let

$$
A_{n}=\{x \in A ; n(x)=n\} .
$$

Since $A$ with the restricted metric $\rho /(A \times A)$ is a complete metric space and

$$
A=\bigcup_{n=1}^{\infty} A_{n}
$$

there is a positive integer $k$ such that the set $A_{k}$ is of the second category in $A$. Consequently, the interior (in the space $A) \operatorname{int}_{A}\left(\operatorname{cl}\left(A_{k}\right)\right)$ of the closure $\operatorname{cl}\left(A_{k}\right)$ of the set $A_{k}$ is nonempty and there is an open set $J$ such that

$$
\emptyset \neq \operatorname{cl}\left(A_{k} \cap J\right)=\operatorname{cl}(J \cap A) .
$$

Consider a function $f_{m}$ with $m>k$. Since the graph $G\left(f_{m} / A\right)$ of the restricted function $f_{m} / A$ is closed, the set $D\left(f_{m} / A\right)$ of all discontinuity points of the restricted function $f_{m} / A$ is nowhere dense in $A$ (see $[1,7]$ ). So there is an open set $I \subset J$ such that

$$
I \cap A \neq \emptyset \text { and } \operatorname{Osc}_{I \cap A} f_{m}<\frac{\eta}{4}
$$

and the restricted function $f_{m} / A$ is continuous at every point of the set $I \cap A$. For each positive integer $j>k$ and for each point $x \in I \cap A_{k}$ the inequality $\left|f_{j}(x)-f(x)\right|<\frac{\eta}{8}$ is true. So, for $j>k$ and $x \in I \cap A_{k}$ we obtain

$$
\left|f_{j}(x)-f_{m}(x)\right| \leq\left|f_{j}(x)-f(x)\right|+\left|f(x)-f_{m}(x)\right|<\frac{\eta}{8}+\frac{\eta}{8}=\frac{\eta}{4} .
$$

Since the restricted function $f_{m} /(I \cap A)$ is continuous and the set $I \cap A_{k}$ is dense in $I \cap A$, the restricted functions $f_{j} /(I \cap A), j>k$, must be also continuous. 
Of course, if $u \in I \cap A$ is a point then, by the continuity of $f_{m} /(I \cap A)$ at $u$, there is an open set $J \subset I$ such that $u \in J$ and $f_{m} /(J \cap A)$ is bounded. Consequently, for $j>k$ the functions $f_{j} /\left(J \cap A_{k}\right)$ are bounded. Since the set $\left.J \cap A_{k}\right)$ is dense in $J \cap A$, the restricted functions $f_{j}(J \cap A), j>k$, are bounded and consequently continuous on $J \cap A$.

Moreover for $x, y \in I \cap A$ and for $j>k$ the inequality

$\left|f_{j}(x)-f_{j}(y)\right| \leq\left|f_{j}(x)-f_{m}(x)\right|+\left|f_{m}(x)-f_{m}(y)\right|+\left|f_{m}(y)-f_{j}(y)\right|<\frac{\eta}{4}+\frac{\eta}{4}+\frac{\eta}{4}=\frac{3 \eta}{4}$

is true. We will show that $\operatorname{osc}_{I \cap A} f \leq \frac{3 \eta}{4}<\eta$. If $\operatorname{osc}_{I \cap A} f>\frac{3 \eta}{4}$ then there are points $u, v \in I \cap A$ such that $|f(u)-f(v)|>\frac{3 \eta}{4}$. But

$$
|f(u)-f(v)|=\left|\lim _{j \rightarrow \infty} f_{j}(u)-\lim _{j \rightarrow \infty} f_{j}(v)\right|=\lim _{j \rightarrow \infty}\left|f_{j}(u)-f_{j}(v)\right| \leq \frac{3 \eta}{4},
$$

and this contradiction finishes the proof.

Now we will describe the discrete convergence of sequences of functions with closed graphs.

A sequence of functions $f_{n}: X \rightarrow \mathcal{R}$ discretely converges to a function $f([4])$ if for each point $x \in X$ there is a positive integer $n(x)$ such that $f_{n}(x)=f(x)$ for $n>n(x)$.

It is known $([4,8,9])$ that a function $f: X \rightarrow \mathcal{R}$ defined on a separable complete metric space $X$ is the discrete limit of a sequence of continuous functions $f_{n}: X \rightarrow \mathcal{R}$ if and only if for each nonempty closed set $A \subset X$ there is a nonempty open set $G \subset X$ such that $G \cap A \neq \emptyset$ and the restricted function $f /(G \cap A)$ is continuous.

Theorem 2. Let $(X, \rho)$ be a separable complete metric space. If a function $f: X \rightarrow \mathcal{R}$ is the discrete limit of a sequence of functions $f_{n}: X \rightarrow \mathcal{R}$ with closed graphs then $f$ is the discrete limit of a sequence of real continuous functions defined on $X$.

Proof. Let $A \subset X$ be a nonempty perfect set. For each point $x \in A$ there is a positive integer $n(x)$ such that $f_{n}(x)=f(x)$ for $n>n(x)$. For $n=1,2, \ldots$ put

$$
A_{n}=\{x \in A ; n(x)=n\} .
$$

Since $(A, \rho /(A \times A))$ is a complete space and

$$
A=\bigcup_{n=1}^{\infty} A_{n},
$$


there is an integer $k>0$ such that the set $A_{k}$ is of the second category in $A$. So there is an open set $J \subset X$ such that

$$
J \cap A \neq \emptyset \text { and } \operatorname{cl}\left(A_{k} \cap J\right)=\operatorname{cl}(A \cap J) .
$$

Consider a function $f_{m}$ with $m>k$. The set $D\left(f_{m} /(I \cap A)\right)$ of discontinuity points of $\left.f_{m} /(J \cap A)\right)$ is nowhere dense in $J \cap A$, so there is an open set $I \subset J$ such that $I \cap A \neq \emptyset$ and the restricted function $f_{m} /(I \cap A)$ is continuous. Since the graphs $G\left(f_{n} / A\right)$ are closed and for $j>k$ we have

$$
f_{j}(x)=f_{m}(x) \text { for } x \in A_{k} \cap I \text { and } \operatorname{cl}(I \cap A)=\operatorname{cl}\left(I \cap A_{k}\right),
$$

the restricted functions $f_{j} /(I \cap A), j>k$, are continuous and

$$
f_{j} /(I \cap A)=f_{m} /(I \cap A) .
$$

Consequently, the restricted function $f /(I \cap A)=f_{m} /(I \cap A)$ is continuous and the proof is completed.

Now we consider the transfinite convergence. Let $\omega_{1}$ be the first uncountable ordinal. We will say [12]) that a transfinite sequence of functions $f_{\alpha}: X \rightarrow \mathcal{R}, \alpha<\omega_{1}$, converges to a function $f$ if for each point $x \in X$ there is a countable ordinal $\beta(x)$ such that $f_{\alpha}(x)=f(x)$ for countable ordinals $\alpha>\beta(x)$.

In the proof of next theorem we will apply the following lemma.

Lemma 1. Let $(X, \rho)$ be a separable complete metric space and let $F \subset X$ be a nonempty closed set. If a transfinite sequence of functions $f_{\alpha}: X \rightarrow \mathcal{R}$, $\alpha<\omega_{1}$, with closed graphs converges to a function $f$ then there are an open set $U$ with $U \cap F \neq \emptyset$ and a countable ordinal $\beta$ such that

$$
f_{\alpha}(x)=f(x) \text { for } x \in U \cap F \text { and } \omega_{1}>\alpha>\beta .
$$

Proof. There is a countable set $A \subset F$ such that $\operatorname{cl}(A)=F$. Let $\beta<\omega_{1}$ be an ordinal such that

$$
f_{\alpha}(x)=f(x) \text { for } x \in A \text { and } \omega_{1}>\alpha>\beta .
$$

Since the graphs of restricted functions $f_{\alpha} / F$ are closed in the product space $F \times \mathcal{R}$, there is an open and dense in $F$ subset $B \subset F$ such that

$$
f_{\alpha}(x)=f(x) \text { for } x \in B \text { and } \omega_{1}>\alpha>\beta .
$$


As an example of such open set $B$ we can take the interior (in $F$ ) of the set of all continuity points of the restricted function $f_{\beta+1} / F$.

Let $B=U \cap F$, where $U$ is open in $X$. Then the set $U$ satisfies all requirements and the proof is completed.

Theorem 3. Let $(X, \rho)$ be a separable complete metric space. If a transfinite sequence of functions $f_{\alpha}: X \rightarrow \mathcal{R}, \alpha<\omega_{1}$, with closed graphs converges to a function $f$ then there is a countable ordinal $\beta$ such that $f_{\alpha}=f$ for all countable ordinals $\alpha>\beta$.

Proof. Let $\mathcal{B}$ be a countable basis of open sets in $X$. By the above Lemma and the transfinite induction we find a transfinite sequence of open sets $U_{\alpha} \in \mathcal{B}, \alpha<\alpha_{0}$, and a transfinite increasing sequence of countable ordinals $\beta(\alpha), \alpha<\alpha_{0}$, such that

$$
\begin{gathered}
X=\bigcup_{\alpha<\alpha_{0}} U_{\alpha}, \\
V_{\alpha}=U_{\alpha} \backslash \bigcup_{\beta<\alpha} U_{\beta} \neq \emptyset
\end{gathered}
$$

and

$$
f_{\beta}(x)=f(x) \text { for } x \in V_{\alpha} \text { and } \beta>\beta(\alpha) .
$$

Since $\alpha_{0}$ is a countable ordinal, there is a countable ordinal $\gamma>\beta(\alpha)$ for all $\alpha<\alpha_{0}$. Obviously, $f_{\alpha}=f$ for all countable $\alpha>\gamma$ and the proof is completed.

The referee observed the following direct proof of Theorem 3 not needing any metric.

It is assumed that the space $X$ has a countable base of open sets. By assumptions, the graph $G(f)$ of the function $f$ is the increasing union of closed sets

$$
G_{\beta}=\left\{(x, y) \in X \times \mathcal{R} ; \forall_{\alpha \geq \beta} f_{\alpha}(x)=y\right\} \text {, where } \beta<\omega_{1} .
$$

As $X \times \mathcal{R}$ has a countable base there is $\beta<\omega_{1}$ such that $G(f)=G_{\beta}$ and hence $f=f_{\alpha}$ for all $\alpha \geq \beta$.

Now we consider the pointwise and discrete convergence of sequences of functions with closed graphs in the case of the density topology.

A point $x \in \mathcal{R}$ is said an outer density point of a set $A \subset \mathcal{R}$ if

$$
\lim _{h \rightarrow 0^{+}} \frac{\mu_{e}([x-h, x+h] \cap A)}{2 h}=1,
$$


where $\mu_{e}$ denotes the Lebesgue outer measure on $\mathcal{R}$.

If a set $A \subset \mathcal{R}$ is measurable (in the Lebesgue sense) then each outer density point of $A$ is said a density point of $A$.

The family $T_{d}$ of all measurable sets $A \subset \mathcal{R}$ such that each point $x \in A$ is a density point of $A$, is a topology said the density topology $([3,13])$. The space $\left(\mathcal{R}, T_{d}\right)$ is completely regular but it is not normal $([13])$.

Now we will consider functions $f:\left(\mathcal{R}, T_{d}\right) \rightarrow\left(\mathcal{R}, T_{e}\right)$.

Theorem 4. If the graph $G(f)$ of a function $f: \mathcal{R} \rightarrow \mathcal{R}$ is closed in the product topology $T_{d} \times T_{e}$ then $f$ is measurable.

Proof. By Davies lemma from [5] it suffices to show that for each measurable set $A \subset \mathcal{R}$ of positive measure and for each positive real $\eta$ there is a measurable set $B \subset A$ of positive measure such that $\operatorname{osc}_{B} f \leq \eta$.

Suppose, on the contrary, that there are a real $\eta>0$ and a measurable set $A \subset \mathcal{R}$ such that $\mu(A)>0$ and $\operatorname{osc}_{B} f>\eta$ for every measurable subset $B \subset A$ of positive Lebesgue measure $\mu(B)$.

There is a closed interval $[c, d]$ such that

$$
d-c<\frac{\eta}{2} \text { and } \mu_{e}\left(f^{-1}([c, d]) \cap A\right)>0 .
$$

Let $H \in T_{d}, H \subset A$, be a nonempty set such that every measurable set $B \subset H \backslash f^{-1}([c, d])$ is of measure zero. As $f$ has a large oscillation on the set $H$, there is a point $x \in H$ with $f(x) \in \mathcal{R} \backslash[c, d]$. Let

$$
y=\sup \left\{\inf _{B} f ; B \subset A \cap f^{-1}([c, d]) \text { and } x \text { is an outer density point of } B\right\} .
$$

Obviously

$$
y \in[c, d] \text { and }(x, y) \in \mathcal{R}^{2} \backslash G(f) .
$$

We will show that $(x, y) \in \operatorname{cl}(G(f))$ with respect to $T_{d} \times T_{e}$. For this let a set $U \in T_{d}$ and an open interval $V$ be such that $x \in U$ and $y \in V$. From the definition of $y$ it follows that there is a set $B \subset f^{-1}([c, d])$ such that $x$ is an outer density point of $B$ and $\inf _{B} f \in V$. Then $x$ is also an outer density point of the set $B \cap U$ and

$$
\inf _{B} f \leq \inf _{B \cap U} f \leq y
$$

Consequently, $\inf _{B \cap U} f \in V$ and there is a point $u \in U \cap B$ with $f(u) \in V$.

So, $(x, y) \in \operatorname{cl}(G(f))$ relative to the topology $T_{d} \times T_{e}$ and the graph $G(f)$ is not closed. This contradiction finishes the proof. 
Since measurable functions are almost everywhere approximately continuous and the sets of measure zero are nowhere dense and closed in the density topology $T_{d}$, we obtain:

Corollary 1. If the graph of a function $f: \mathcal{R} \rightarrow \mathcal{R}$ is closed in the product topology $T_{d} \times T_{e}$ then the set $D(f)$ of all $T_{d}$-discontinuity points of $f$ is closed and nowhere dense in $T_{d}$.

Functions $f: \mathcal{R} \rightarrow \mathcal{R}$ with closed graph in the topology $T_{d} \times T_{e}$ may be nonborelian.

\section{Example.}

Let $C$ be the ternary Cantor set and let $\left(I_{n}\right)_{n}$ be an enumeration of all components of the complement $\mathcal{R} \backslash C$ such that $I_{n} \cap I_{m}=\emptyset$ for $m \neq n$. Let $B \subset C$ be a nonborelian set. For $n=1,2, \ldots$ let $f_{n}: I_{n} \rightarrow[n, \infty)$ be a continuous function such that if $x$ is an endpoint of $I_{n}$ then $\lim _{I_{n} \ni t \rightarrow x} f(t)=$ $\infty$. Then the graph of the function

$$
f(x)=\left\{\begin{array}{cll}
f_{n}(x) & \text { for } & x \in I_{n}, n \geq 1 \\
1 & \text { for } & x \in B \\
0 & \text { for } & x \in C \backslash B
\end{array}\right.
$$

is closed in the product topology $T_{d} \times T_{e}$, but $f$ is non-Borel.

Theorem 5. If $f: \mathcal{R} \rightarrow \mathcal{R}$ is measurable then there is a sequence of functions $g_{n}: \mathcal{R} \rightarrow \mathcal{R}$ with closed graphs in the topology $T_{d} \times T_{e}$ which discretely converges to $f$.

Proof. By Lusin Theorem there are closed (in $T_{e}$ ) sets $A_{n}, n \geq 1$, such that the restricted functions $f / A_{n}$ are $T_{e}$-continuous,

$$
A_{n} \subset A_{n+1} \text { for } n=1,2, \ldots \text { and } \mu_{e}\left(\mathcal{R} \backslash \bigcup_{n=1}^{\infty} A_{n}\right)=0 \text {. }
$$

The set

$$
A=\mathcal{R} \backslash \bigcup_{n=1}^{\infty} A_{n}
$$

is an $G_{\delta}$-set of measure zero. So for each integer $n \geq 1$ there is an $G_{\delta}$-set $E_{n} \supset A$ of measure zero which contains all endpoints of components of the complement $\mathcal{R} \backslash A_{n}$. By Zahorski Lemma ([3]) for $n \geq 1$ there are approximately continuous functions $f_{n}: \mathcal{R} \rightarrow[0,1]$ (i.e. $f_{n}$ are continuous as applications from $\left(\mathcal{R}, T_{d}\right)$ to $\left.\left(\mathcal{R}, T_{e}\right)\right)$ such that $f_{n}(x)=0$ for $x \in E_{n}, f_{n}(x)>0$ otherwise on $\mathcal{R}$ and $f_{n}$ are $T_{e}$-continuous at points $x \in E_{n}$. 
Let $\left(I_{k, n}\right)_{k}$ be an enumeration of all components of the complement $\mathcal{R} \backslash A_{n}$ such that $I_{k} \cap I_{j}=\emptyset$ for $k \neq j$. For $n \geq 1$ define

$$
g_{n}(x)=\left\{\begin{array}{cll}
f(x) & \text { for } & x \in A_{n} \cup E_{n} \\
\max \left(k, \frac{1}{f_{n}(x)}\right) & \text { for } & x \in I_{k, n} \backslash E_{n}, k \geq 1 .
\end{array}\right.
$$

Then the graphs $G\left(g_{n}\right)$ are closed in $T_{d} \times T_{e}$ for $n \geq 1$ and the sequence $\left(g_{n}\right)_{n}$ discretely converges to $f$.

Remark 1. Since the pointwise limit $f$ of a sequence of approximately continuous functions $f_{n}: \mathcal{R} \rightarrow \mathcal{R}$ is of the second Baire class and since there are nonborelian measurable functions, Theorems 1 and 2 are not true for the case the topology $T_{d} \times T_{e}$.

Theorem 6. Assume that Continuum Hypothesis (CH) is true. For each function $f: \mathcal{R} \rightarrow \mathcal{R}$ there are functions $f_{\alpha}: \mathcal{R} \rightarrow \mathcal{R}$ with closed graphs in the topology $T_{d} \times T_{e}$, where $\alpha<\omega_{1}$, such that the transfinite sequence $\left(f_{\alpha}\right)_{\alpha<\omega_{1}}$ converges to a function $f$.

Proof. Enumerate all reals in a transfinite sequence $\left(a_{\alpha}\right)_{\alpha<\omega_{1}}$ such that $a_{\alpha} \neq a_{\beta}$ for $\alpha \neq \beta$.

For $\alpha<\omega_{1}$ let

$$
A_{\alpha}=\left\{a_{\beta} ; \beta<\alpha\right\}
$$

and let $g_{\alpha}: \mathcal{R} \rightarrow[0,1]$ be an approximately continuous function such that

$$
\mu_{e}\left(g_{\alpha}^{-1}(0)\right)=0 \text { and } A_{\alpha} \subset g_{\alpha}^{-1}(0),
$$

and $g_{\alpha}$ is continuous at each point $x$ at which $g_{\alpha}(x)=0$. Then the function

$$
f_{\alpha}(x)=\left\{\begin{array}{ccl}
\frac{1}{g_{\alpha}(x)} & \text { if } & g_{\alpha}(x) \neq 0 \\
f(x) & \text { otherwise on } & \mathcal{R}
\end{array}\right.
$$

has the closed graph $G\left(f_{\alpha}\right)$ in the topology $T_{d} \times T_{e}$ and

$$
f_{\alpha}(x)=f(x) \text { for } x \in A_{\alpha} .
$$

Evidently, the transfinite sequence $\left(f_{\alpha}\right)_{\alpha<\omega_{1}}$ converges to $f$. This completes the proof.

In connection with the last theorem remember ([11]) that a function $f$ : $\mathcal{R} \rightarrow \mathcal{R}$ is the limit of a transfinite sequence of approximately continuous functions $f_{\alpha}: \mathcal{R} \rightarrow \mathcal{R}$ if and only if it is of the first Baire class. 


\section{References}

[1] Baggs I.; Functions with a closed graphs, Proc. Amer. Math. Soc. 43 (1974), 439-442.

[2] Borsík J., Doboš J. and Repický M.; Sums of quasicontinuous functions with closed graphs, Real Analysis Exchange 25 No. 2 (1999-2000), 679690.

[3] Bruckner A.M.; Differentiation of real functions, Lectures Notes in Math. 659, Springer-Verlag, Berlin 1978.

[4] A. Császár and M. Laczkovich; Discrete and equal convergence, Studia Sci. Math. Hungar. 10 (1975), 463-472.

[5] Davies R. O.; Approximate continuity implies measurability, Math. Proc. Camb. Philos. Soc. 73 (1973), 461-465.

[6] Doboš J.; A note on the functions the graph of which are closed sets, Acta Math. Univ. Comenian. 40-41 (1982), 285-288.

[7] Doboš J.; On the set of points of discontinuity for functions with closed graphs, Cas. pest. mat. 110 (1985), 60-68.

[8] Grande Z.; Baire functions and their restrictions to special sets, Math. Slovaca 43 (1993), 447-453.

[9] Kirchheim B.; Baire one star functions, Real Analysis Exchange 8 (1992//93), 385-389.

[10] Kostyrko P.; A note on the functions with closed graphs, Cas. pest. mat. 94 (1969), pp. 202-205.

[11] Lipiński J. S.; On transfinite sequences of mappings, Cas. pest. mat. 101 (1976), 153-158.

[12] Sierpiński W.; Sur les suites transfinies convergentes de fonctions de Baire, Fund. Math. 1 (1920), 132-141.

[13] Tall F. D.; The density topology, Pacific J. Math. 62 (1976), pp.275-284. 
